\section{An Antimicrobial Cerebroside from the Liposoluble Constituent of Cervus Nippon Antler Velvet Layer}

\author{
Nina Bao ${ }^{1 * *}$, Yongguang Yin ${ }^{2}$ and Panpan Wang ${ }^{3}$ \\ ${ }^{1}$ School of Biological and Food Engineering, Suzhou University, Suzhou 234000, China \\ ${ }^{2}$ School of Biological and Agricultural Engineering, Jilin University, Changchun \\ 130022, China \\ ${ }^{3}$ School of Foreign Studies, Suzhou University, Suzhou 234000, China
}
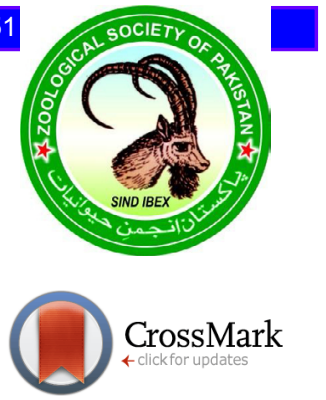

\begin{tabular}{l} 
Article Information \\
Received 23 May 2018 \\
Revised 28 June 2018 \\
Accepted 16 July 2018 \\
Available online 03 August 2018 \\
Authors' Contribution \\
\hline NB designed the study, performed the \\
experimental work, analyzed the data \\
and wrote the article. YY helped in \\
the funds of this study. PW helped in \\
writing. \\
Key words \\
Cerebroside, Antler velvet layer, TLC, \\
IR, Bioactivity.
\end{tabular}

\section{INTRODUCTION}

$\mathrm{A}^{5}$ s one kind of traditional Chinese medicine, Cervus nippon antler velvet layer has been widely used in oriental medicine (Zhou and Li, 2009a). The liposoluble constituent of cervus nippon antler velvet layer (sex hormone (Zhou and Li, 2009a; Zhou et al., 2009a), phospholipids, p-hydroxybenzaldehyde (Zhou and Li, 2009b; Zhou et al., 2009b), uracil, hypoxanthine (Zhou et al., 2009b)) which plays a significant role in sexualreinforcing (Chen et al., 1992), anti-aging effect (Zhang et al., 1992; Wang et al., 1988), and so on is also been widely investigated. But glycolipid (Inagaki et al., 2006; Para et al., 2009; Mansoor et al., 2007; La et al., 2012; Ikada et al., 2009) was scarce investigated which also belonged to the liposoluble constituent of cervus nippon antler velvet layer.

Glycolipid (cerebroside and ganglioside), particularly cerebroside, has been proved to exhibit various physiological activities, including antitumor/cytotoxic (Natori et al., 1994; Jin et al., 1994; Li et al., 1995; Chen et al., 2009), antifungal (Jin et al., 1994), immunomodulatory, cyclooxygenase inhibitory, and antifouling activities (Mansoor et al., 2007). Thus, it caused me great interest in finding the physiological activities of cerebroside from the liposoluble constituent of cervus nippon antler velvet layer.

\footnotetext{
Corresponding author: ninabao1984@163.com 0030-9923/2018/0005-1747 \$ 9.00/0

Copyright 2018 Zoological Society of Pakistan
}

Generally, using chloroform and methanol to extract cerebroside from the tissues will cause losses in biological activity, so we used supercritical carbon dioxide to extract the liposoluble constituent of cervus nippon antler velvet layer and extracted cerebroside with petroleum ether. Meanwhile, silica gel column was used to purify cerebroside and thin layer chromatography (TLC) was used to identified cerebroside. Characterization of extraction was analyzed by IR (Chen et al., 2003; Cateni et al., 2003).

The aims of this paper were to (i) use petroleum ether to extract cerebroside from the liposoluble constituent of cervus nippon antler velvet layer, (ii) use silica gel column to isolate and purify cerebroside, (iii) use IR to characterize cerebroside and (iv) use agar diffusion test to evaluate antimicrobial properties.

\section{MATERIALS AND METHODS}

\section{Materials}

Development College of Agriculture University provided the boiled-dry cervus nippon antler velvet. More than $99.0 \%$ purity of the cholesterol standard sample was purchased from the Chemical Company of Sigma (USA).

\section{Supercritical $\mathrm{CO}_{2}$ extraction}

Speed SFE instrument was used to execute the supercritical extraction which was made by Applied Separations Incorporated, Allenton, PA, USA. The 
different factors of supercritical carbon dioxide extraction experiment were used to extract the liposoluble constituent of cervus nippon antler velvet layer as follows: the size of particle $250 \mu \mathrm{m}$, co-solvent ethanol-water $(75: 25, \mathrm{v} / \mathrm{v})$, temperature of extraction $70^{\circ} \mathrm{C}$, and pressure of extraction $30 \mathrm{MPa}$.

\section{Petroleum ether extraction}

Weighed five grams of the liposoluble constituent of cervus nippon antler velvet layer, and stirred them to dissolve in the petroleum ether completely. After staying a moment, the cerebroside was obtained by centrifuge at $6000 \times \mathrm{g}$. The residue obtained by centrifuge was heated for $6 \mathrm{~h}$ under the temperature of $40^{\circ} \mathrm{C}$ in vacuo which turned to be a power with amorphism.

\section{Determination of cholesterol}

The method of O-phthalaldehyde colorimetric which was described by Xu et al. (2006) was used to determine cholesterol contents of the residue.

\section{Separation and purification of cerebroside}

The dissolved cerebroside which was obtained by using chloroform and methanol (2:1/v:v) was subjected to column chromatography on silica gel, and then was eluted with chloroform-methanol- $\mathrm{H}_{2} \mathrm{O}(20: 1: 0.2)$, by which 3 fractions $(1,2,3)$ was afforded. Fraction 2 was isolated again to yield cerebrosides by column chromatography on silica gel, and with the method of eluting with chloroform and chloroform-methanol (19:1/v:v), respectively. Chloroform and chloroform-methanol $(19: 1 / \mathrm{v}: \mathrm{v})$ as a solvent to purify the cerebrosides by using column chromatography on silica gel, they were identified by TLC (Cateni et al., 2010; Ravneet et al., 2010).

\section{Identification of cerebroside}

The purified cerebroside was evaluated with TLC. The TLC plates were purchased from the Chemical Company of Qingdao (China). 20 microlitres solution was applied and used chloroform:methanol: water $=10: 1: 0.2$ $(\mathrm{v} / \mathrm{v})$ as the solvent system.

\section{Characterization analysis of cerebroside}

Using $\mathrm{KBr}$ to preparing the sample as pellet, and then scanned it from $4000 \mathrm{~cm}^{-1}$ to $500 \mathrm{~cm}^{-1}$ by IRPrestige- 21 infrared spectrophotometer, the peaks of which was reported in $\mathrm{cm}^{-1}$.

\section{Antimicrobial properties of cerebroside}

Antimicrobial properties of cerebroside were evaluated by $E$. coli which was got from excrement with the method of the minimum inhibitory concentration (MIC) (Cateni et al., 2010; Ravneet et al., 2010; Liaqat et al., 2017). DMSO $(20 \%)(1 \mathrm{~mL})$ was used to dissolve dried extraction and purified compound, and obtain a concentration of 8000 and $7000 \mu \mathrm{g} / \mathrm{mL}$ finally. $500 \mu \mathrm{L}$ microbial suspension which grew at $37^{\circ} \mathrm{C}$ for an overnight was added to the tube one by one, and subsequently cultured aerobically $24 \mathrm{~h}$ under the temperature of $37^{\circ} \mathrm{C}$. MIC will be proved to the lowest concentration of the cerebroside if there were color change (red to yellow) or visible growth because with the microbial growth, it will form acidic metabolites.

\section{RESULTS AND DISCUSSION}

\section{Effect of liquid-to-solid ratio on the weight of residue}

The liquid-to-solid ratio, which directly influences the purity of crude cerebrosideis, has great effect on the residue of cerebroside extraction. As shown in Figure 1, the amount of residue of cerebroside extraction decreased from $0.402 \pm 0.007 \mathrm{~g}$ to $0.076 \pm 0.016 \mathrm{~g}$ when the liquid-tosolid ratio increased from 5 to 19 . The cholesterol which was retained in crude cerebroside varied from $0.017 \pm 0 \mathrm{~g}$ to $0.008 \pm 0 \mathrm{~g}$. This is so obvious that the more petroleum ether the less residue, which is because more petroleum ether caused more dissolution of fatty acid which resulted in less residue, therefore made crude cerebroside more pure. When the liquid-to-solid ratio changed from 15 to 19 , the weight of cerebroside decreased steadily, this indicated that the free fat acid almost dissolves in petroleum ether. The present study chose the liquid-to-solid ratio at 15 to extract the cerebroside.

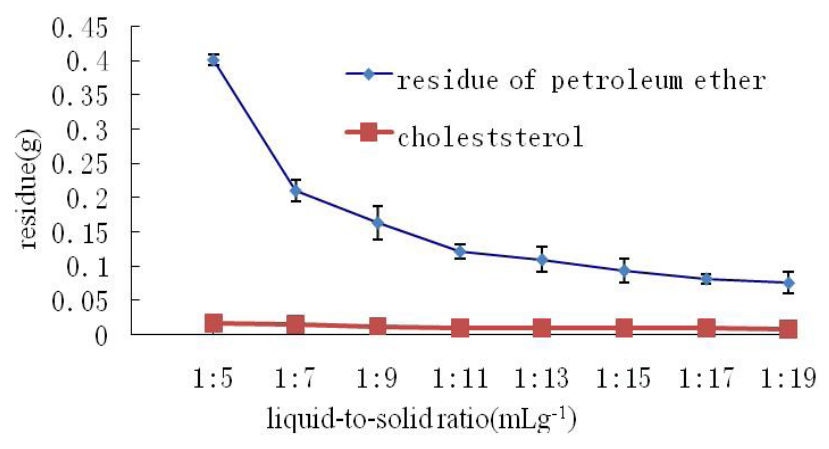

Fig. 1. Effect of liquid-to-solid ratio on the weight of residue.

\section{Effect of standing time on the weight of residue}

Standing time also plays a significant role for the residue of cerebroside extraction. Figure 2 revealed that as the standing time changed from $15 \mathrm{~min}$ to 75 min, cerebroside decreased from $0.108 \pm 0.010 \mathrm{~g}$ to $0.086 \pm 0.001 \mathrm{~g}$. The cholesterol which was retained in crude cerebroside varied from $0.010 \pm 0.002 \mathrm{~g}$ to $0.011 \pm 0 \mathrm{~g}$, with 
little variation with the standing time. When the standing time reached $45 \mathrm{~min}$, the weight of cerebroside changed little and the curve became smooth. With the increase of standing time, it can be seen that more fatty acid dissolves in petroleum ether and reach saturation. So the weight of cerebroside will decrease and then turn to be constant. The weight of cerebroside decreases until it comes to $45 \mathrm{~min}$, therefore this study chosen standing time at $45 \mathrm{~min}$ as a precondition to carry on the experiment.

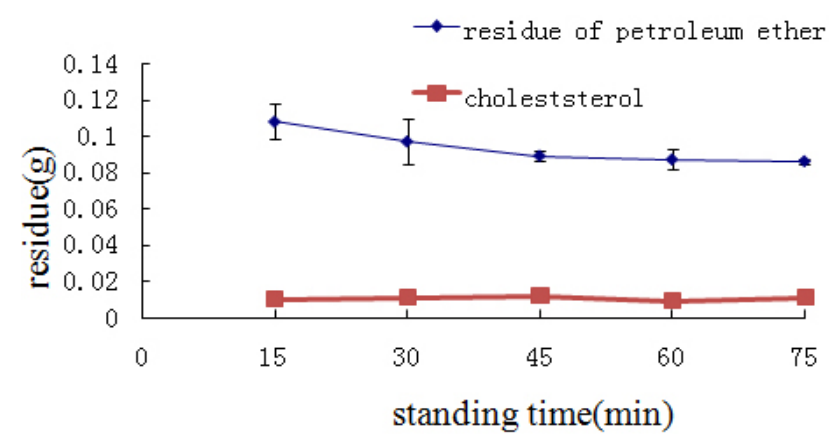

Fig. 2. Effect of standing time on the cerebroside extraction.

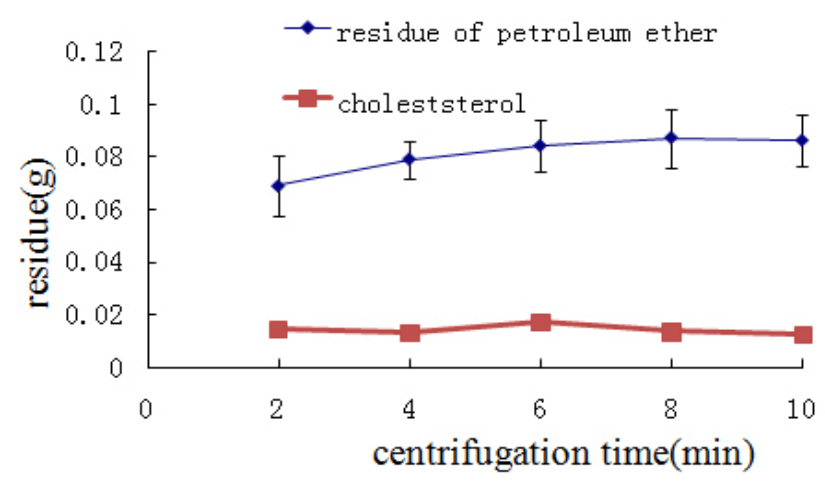

Fig. 3. Effect of centrifugation time on the cerebroside extraction.

\section{Effect of centrifugation time on the weight of residue}

Figure 3 revealed that centrifugation time affects the weight of residue. As the time increases from $2 \mathrm{~min}$ to $10 \mathrm{~min}$, the weight of cerebroside increases from $0.069 \pm 0.011 \mathrm{~g}$ to $0.086 \pm 0.010 \mathrm{~g}$. The cholesterol which was retained in crude cerebroside varied from $0.015 \pm 0.001 \mathrm{~g}$ to $0.013 \pm 0.001 \mathrm{~g}$. It's true that the more centrifugation time the more weight of cerebroside, when it comes to $6 \mathrm{~min}$, the change trend of the cerebroside weight becomes smooth. Therefore, the centrifugation time at $6 \mathrm{~min}$ was favorable for this study.

\section{Identification of cerebroside}

Figure 4 indicated that TLC was used to identify the residue, which was extracted with petroleum ether. Ethanol$\mathrm{H}_{2} \mathrm{SO}_{4}$-spray method was adopted to visual the cerebroside and GF $254(20 \times 20)$ was used to carry out TLC. $5 \%$ aq. $\mathrm{H}_{2} \mathrm{SO}_{4}$ was firstly used to spray the sample, which then was heated at $110^{\circ} \mathrm{C}$ for $5 \mathrm{mins}$ until the brownish yellow trace appeared on the white background. Lane 1 was crude cerebroside, Lane 2 was eluted with chloroform, while Lane 3 was eluted with chloroform-methanol 19:1, and Lane 4 stood for the cholesterol standards. One spot means pure on silica gel plate of TLC. The results showed that when chloroform was used to elute, only cholesterol can be obtained, while chloroform-methanol 19:1 was adopted to elute, only cerebroside can be acquired which signifies the purify of cerebroside.

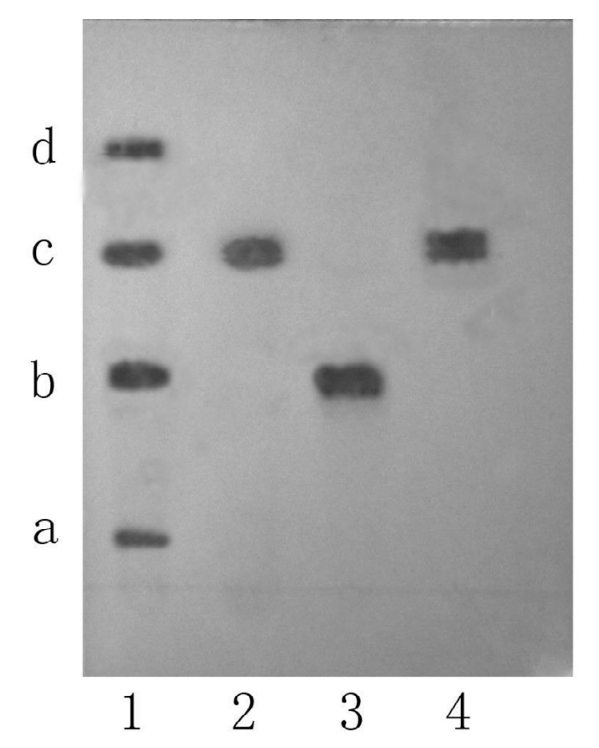

Fig. 4. TLC of cerebroside in the extracts. 1, the crude cerebrosdie; 2 and 3, constituent eluted with chloroform and chloroform-methanol (19:1/v:v), respectively; 4, standard of cholesterol.

\section{IR analysis of cerebroside}

IR absorbance peaks at $3311 \mathrm{~cm}^{-1}, 1642 \mathrm{~cm}^{-1}$ and 1550 $\mathrm{cm}^{-1}$ (Fig. 5) were in consistent with absorbance peaks of hydroxyl and amide groups.

As the infrared characteristic absorption peaks of cerebroside are hydroxyl and amide groups, it is possible to infer cerebroside. The IR absorptions which reported by Chen et al. (2003) showed that hydroxyl and amide groups were $3323 \mathrm{~cm}^{-1}, 1661 \mathrm{~cm}-1$ and $1557 \mathrm{~cm}^{-1}$, respectively; and as reported by Cateni et al. (2003), the IR absorbance peaks of hydroxyl and amide groups were $3413 \mathrm{~cm}^{-1}, 1646 \mathrm{~cm}^{-1}$ and $1540 \mathrm{~cm}^{-1}$, respectively. In conclusion, residue which was extracted from the liposoluble constituent of cervus nippon antler velvet layer was confirmed to be cerebroside. 


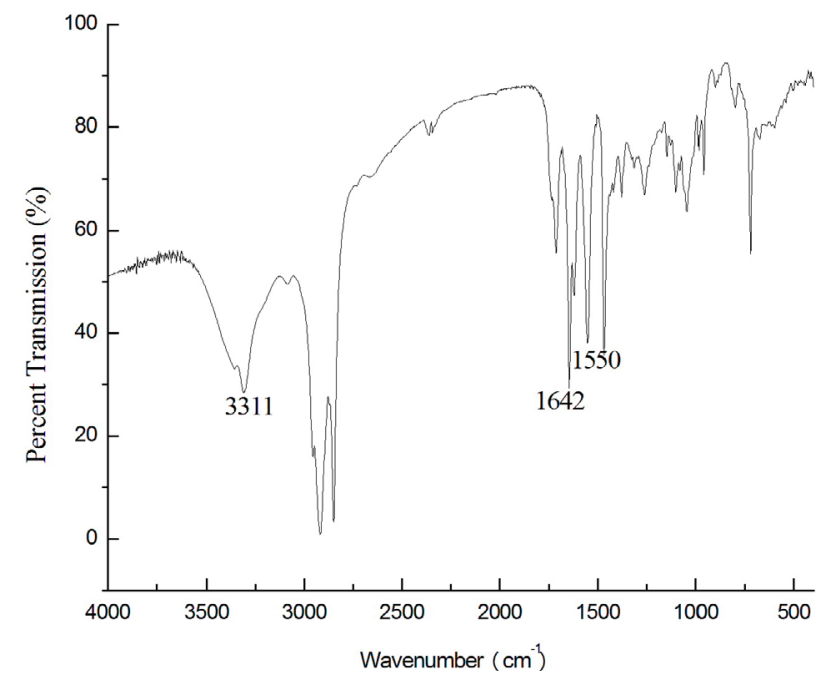

Fig. 5. IR spectrum of cerebroside.

\section{Antimicrobial properties}

The microbroth dilutioin method (against Staphylococcus aureus and Escherichia coli) was used to evaluate the biological activity of the cerebroside which was extracted from the liposoluble constituent of cervus nippon antler velvet layer. Results showed that cerebroside inhibited growth of E. coli. Meanwhile the minimum inhibitory concentrations of the purified one and the crude one were $27 \mu \mathrm{g} / \mathrm{mL}$ and $125 \mu \mathrm{g} / \mathrm{mL}$, respectively.

The known cerebrosides isolated from Euphorbia peplis L. and Pinellia ternate showed good antimicrobial activities on Gram positive bacterium (S. aureus), both of them were not inhibitory to the Gram negative bacterium (E. coli). However, the biological activities of cerebroside from cervus nippon antler velvet showed no activity against $S$. aureus, but it worked on E. coli. In conclusion, the purified cerebroside and crude cerebroside isolated from the liposoluble constituent of cervus nippon antler velvet layer showed inhibitory activities on E. coli, but had no biological activities on $S$. aureus. Meanwhile, the purified cerebroside showed better than the crude cerebroside.

\section{CONCLUSIONS}

This study used petroleum ether to investigate the extraction of cerebroside from the liposoluble constituent of cervus nippon antler velvet layer. The liquid-to-solid ratio 15 , standing time $45 \mathrm{~min}$, and centrifugation time $6 \mathrm{~min}$ were the conditions for cerebroside extraction. The cerebroside was purified by column chromatography of silica gel eluting with chloroform and chloroformmethanol (19:1/v:v), which was identified by TLC. IR analysis showed the residue contained hydroxyl and amide groups, which are the characteristic absorption peaks of cerebrosides and can be used to testify the residue, which is cerebroside. The purified cerebroside showed better antimicrobial properties than the crude one, and the MIC of them were $27 \mu \mathrm{g} / \mathrm{mL}$ and $125 \mu \mathrm{g} / \mathrm{mL}$, respectively. The cerebroside had inhibitory activities on E. coli, but had no biological activities on $S$. aureus.

\section{ACKNOWLEDGMENTS}

We acknowledge the financial supports of the Science Foundation of SuZhou University (2015JB09, 2015YKF04).

\section{Statement of conflict of interest}

The authors declare that there is no conflict of interests regarding the publication of this article.

\section{REFERENCES}

Cateni, F., Zilic, J., Falsone, G., Scialino, G. and Banfi, E., 2003. New cerebrosides from Euphorbia peplis L.: Antimicrobial activity evaluation. Bioorg. Med. Chem. Lett., 13: 4345-4350. https://doi. org/10.1016/j.bmcl.2003.09.044

Cateni, F., Zilic, J., Zacchigna, M. and Procida, G., 2010. Cerebrosides with antiproliferative activity from Euphorbia peplis L. Fitoterapia, 81: 97-103. https://doi.org/10.1016/j.fitote.2009.08.022

Chen, J.H., Cui, G.Y., Liu, J.Y. and Tan, R.X., 2003. Pinelloside, an antimicrobial cerebroside from Pinellia ternata. Phytochemist, 64: 903-906. https://doi.org/10.1016/S0031-9422(03)00421-7

Chen, L., Wang, J.J., Song, H.T., Zhang, G.G. and Qin, L.P., 2009. New cytotoxic cerebroside from Gynura divaricata. Chinese Chem. Lett., 20: 1091-1093. https://doi.org/10.1016/j.cclet.2009.04.021

Chen, X.G., Chang, D.Y., Cui, Z.Y. and Wang, B.X., 1992. Effects of the water extract of pilose antler on some biochemical indicators related to aging in old mice. Pharmacol. Clin. Chinese Mat. Med., 8: 17-20.

Inagaki, M., Nakata, T. and Higuchi, R., 2006. Isolation and structure of a galactocerebroside molecular species from the starfish Culcita novaeguineae. Chem. Pharm. Bull., 54: 260-261. https://doi. org/10.1248/cpb.54.260

Ikada, Y., Inagaki, M., Yamada, K., Zhang, X.W., Zhang, B., Miyamoto, T. and Higuchi, R., 2009. Isolation and structure of a galactocerebroside from the sea cucumber Bohadschia argus. Chem. Pharm. Bull., 57: 315-317. https://doi.org/10.1248/cpb.57.315 
Jin, W.Z., Rinehart, K.L. and Jares-Erijman, E.A., 1994. Ophidiacerebrosides: Cytotoxic glycosphingolipids containing a novel sphingosine from a sea star. $J$. Org. Chem., 59: 144-147. https://doi.org/10.1021/ jo00080a023

La, M.P., Shao, J.J., Jiao, J. and Yi, Y.H., 2012. Three cerebrosides from the sea cucumber Cucumaria frondosa. Chinese J. Nat. Med., 10: 105-109. https://doi.org/10.3724/SP.J.1009.2012.00105

Li, H.Y., Matsunaga, S. and Fusetani, N., 1995. Halicylindrosides, antifungal and cytotoxic cerebrosides from the marine sponge Halichondria cylindrata. Tetrahedron, 51: 2273-2280. https:// doi.org/10.1016/0040-4020(94)01097-J

Liaqat, I., Arshad, N., Arshad, M., Mirza, S. A., Ali, N. M. amd Shoukat, A., 2017. Antimicrobial activity of some medicinal plants extracts against food industry isolates. Pakistan J. Zool., 49: 523-530. http://dx.doi.org/10.17582/journal. pjz/2017.49.2.565.572

Mansoor, T.A., Shinde, P.B., Luo, X., Hong, J., Lee, C., Sim, C.J., Son, B.W. and Jung, J.H., 2007. Renierosides, cerebrosides from a marine sponge Haliclona (Reniera) sp. J. Nat. Prod., 70: 14811486. https://doi.org/10.1021/np070078u

Natori, T., Morita, M., Kohji, A. and Koezuka, Y., 1994. Agelasphins, novel antitumor and immunostimulatory cerebrosides from the marine sponge Agelas mauritianus. Tetrahedron, 50: 2771-2784. https://doi.org/10.1016/S00404020(01)86991-X

Para, T., Mansoor, T.A., Shinde, P.B., Bao, B.Q., Hong, J. and Jung, J.H., 2009. New cerebrosides from a marine sponge Haliclona (Reniera) sp. Chem. Pharm. Bull., 57: 106-111. https://doi.org/10.1248/ cpb.57.106

Ravneet, K., Grewal, A. and Mahmood, K.A., 2010.
The effects of ethanol administration on brush border membrane glycolipids in rat intestine. Alcohol, 44: 515-522. https://doi.org/10.1016/j. alcohol.2010.07.008

Wang, B.X., Zhao, X.H., Yang, X.W., Kaneko S., Hattori, M., Namba, T. and Nomura, Y., 1988. Inhibition of lipid peroxidation of deer antler (Rokujo) extract in vivo and in vitro. J. Med. Pharma. Soc. WakanYaku, 5: 123-128.

$\mathrm{Xu}$, Z.H., Wang, J.Y. and Li, S.F., 2006. Rapid determination of the cholesterol content in the supercritical carbon dioxide extract of deer antler. Chinese Herb. Med., 29: 800-802.

Zhang, Y.Z., Liu, G.L., Chen, X.G. and Bai, S.G., 1992. Effect of sexual hormones on MDA and SOD activity in the brain of old mice. J. Gerontol., 12: 165-166.

Zhou, R. and Li, S.F., 2009a. Supercritical carbon dioxide and co-solvent extractions of estradiol and progesterone from antler velvet. $J . F d$. Comp. Anal., 22: 72-78. https://doi.org/10.1016/j. jfca.2008.07.008

Zhou, R. and Li, S.F., 2009b. In vitro antioxidant analysis and characterisation of antler velvet extract. $F d$. Chem., 114: 1321-1327. https://doi.org/10.1016/j. foodchem.2008.11.010

Zhou, R., Li, S.F. and Zhang, D.C., 2009a. Combination of supercritical fluid extraction with ultrasonic extraction for obtaining sex hormones and IGF1 from antler velvet. Chinese J. Chem. Eng., 17: 373-380. https://doi.org/10.1016/S10049541(08)60219-0

Zhou, R., Wang, J.Y., Li, S.F. and Liu, Y., 2009b. Supercritical fluid extraction of monoamine oxidase inhibitor from antler velvet. Sep. Purif. Technol., 65: 275-281. https://doi.org/10.1016/j. seppur.2008.10.036 